\title{
Evaluación de la susceptibilidad a Macrophomina phaseolina (Tassi) Goid en diferentes variedades e híbridos comerciales de sorgo en Sinaloa, México Evaluation of susceptibility to Macrophomina phaseolina (Tassi) Goid in different varieties and commercial hybrids of sorghum Sinaloa, Mexico
}

Tomás Moreno Gallegos ${ }^{1}$, Claudia Maria Melgoza-Villagómez ${ }^{1}$, Venancio Cuevas Reyes ${ }^{1}$ y Luis Alberto Hernández-Espinal ${ }^{2}$

Palabras Clave: Sorghum bicolor; pudrición carbonosa del tallo; tolerantes

Recepción 19-10-2016 / Aceptación 03-03-2017

\section{Resumen}

El objetivo del presente trabajo fue evaluar la reacción a $M$. phaseolina de tres variedades experimentales y siete variedades de polinización libre del INIFAP, así como 14 híbridos comerciales de sorgo, con la finalidad de identificar variedades e híbridos que puedan presentar tolerancia o menor susceptibilidad a esta enfermedad. Los experimentos se establecieron en Culiacán, Sinaloa, México, durante los ciclos Primavera-Verano de 2010 y 2011, bajo condiciones de campo en temporal. Se utilizó un diseño de bloques al azar en cada experimento con tres repeticiones y un análisis combinado para los dos ciclos agrícolas. La parcela experimental consistió en inocular 10 plantas con mondadientes infectados del hongo. El análisis de varianza detecto diferencias significativas $(\mathrm{p}<0.05)$ en longitud de lesión ocasionada por el hongo entre años. En el ciclo 2010, se observó una longitud de lesión promedio significativamente mayor $(10.10 \mathrm{~cm})$ en comparación con el ciclo $2011(5.79 \mathrm{~cm})$. Los genotipos con menor longitud de lesión en promedio de los dos ambientes fueron: Fortuna y Mazatlán-16 (3.5 y $4.95 \mathrm{~cm}$, respectivamente). Los genotipos con mayor longitud de lesión fueron Kilate, GSTAR-7402 y G-STAR-7205 (13.2, 15.7 y 16.65 cm, respectivamente). No existió correlación entre la longitud de lesión y rendimiento de grano. Las variedades Fortuna y Mazatlán-16 mostraron menor daño a $M$. phaseolina, por lo cual se sugieren para siembras de temporal y como progenitores en la formación de híbridos de sorgo tolerantes a esta enfermedad.

\footnotetext{
Abstract

The aim of the present work was to evaluate the reaction to $M$. phaseolina of three experimental varieties and seven varieties of free pollination of INIFAP, as well as 14 commercial sorghum

${ }^{1}$ Instituto Nacional de Investigaciones Forestales, Agrícolas y Pecuarias. E-mail: cuevas.venancio@gmail.com ${ }^{2}$ Facultad de Medicina Veterinaria y Zootecnia, Universidad Autónoma de Sinaloa

(C) Universidad De La Salle Bajío (México)
} 
hybrids, in order to identify varieties and hybrids that may present tolerance or less susceptibility to this sickness. The experiments were established in Culiacan, Sinaloa, Mexico, during the Spring-Summer cycles in 2010 and 2011, under rainfall field conditions. A randomized block design with three replications and a combined analysis for the two agricultural cycles were used. The experimental plot consisted of 10 plants inoculated with the fungus infected toothpicks. The analysis of the variance showed significant differences $(\mathrm{p}<0.05)$ in lesion length caused by the fungus between years, in the cycle 2010, a length significantly greater mean lesion $(10.10 \mathrm{~cm})$ was observed compared to the 2011 cycle $(5.79 \mathrm{~cm})$. As for the length of the injury caused by the fungus, the genotypes that less lesion length on average of the two environments were: Fortuna and Mazatlán-16 (3.5 and $4.95 \mathrm{~cm}$, respectively). The genotypes with more lesion length were Kilate, G-STAR-7402 and G-STAR-7205 (13.2, 15.7 and $16.65 \mathrm{~cm}$, respectively). There was no correlation between the lesion length and the grain yield. The Fortuna and Mazatlan-16 varieties showed less damage to $M$. phaseolina, for that cause so plantings are suggested for temporary and as parents in the formation of hybrid sorghum tolerant to this disease.

Keywords: Sorghum bicolor; charcoal stalk rot; tolerant. 


\section{Introducción}

En México, la superficie sembrada de sorgo (Sorghum bicolor L. Moench) en 2014 fue de 2, 078, 496 ha, con una producción de 8 millones 394 mil 056 t de grano. Sinaloa ocupa el segundo lugar nacional en superficie sembrada; en 2014 se sembraron 289 mil 286 ha de las cuales más del 70 $\%$ se cultiva bajo condiciones de temporal, que corresponde al ciclo Primavera-Verano. En cuanto a producción, Sinaloa ocupa el tercer lugar en México con un millón 174 mil 599 t de grano al año (SIAP, 2016). Sin embargo, uno de los principales factores que limitan la producción de sorgo granifero es la incidencia de enfermedades, entre ellas, la ocasionada por el hongo Macrophomina phaseolina (Tassi) Goid, que ocasiona la pudrición carbonosa del tallo en sorgo y otros cultivos de importancia económica en México (Dhingra y Sinclair, 1978).

El hongo ataca a más de 500 especies de plantas y cerca de 100 familias (Mihail y Taylor, 1995). La incidencia de esta enfermedad es más severa en regiones donde frecuentemente se presentan períodos largos con temperaturas del suelo mayores a $32^{\circ} \mathrm{C}$, baja humedad del suelo y siembras tardías (Clafin y Giorda, 2002). Las altas temperaturas y la sequía favorecen el desarrollo de la enfermedad (Clafin y Giorda, 2002; Tuinstra et al., 2002; Williams-Alanís et al., 2009).

Se ha reportado que M. phaseolina es uno de los patógenos que prevalece mayor tiempo en el suelo, sobreviviendo principalmente en forma de esclerocios, lo cual constituye la principal fuente de inóculo (Cook et al., 1973; Olaya et al., 1996). La incidencia de la enfermedad en el cultivo varía de un año a otro y de región a región, dependiendo del manejo agronómico y de las condiciones climáticas (Singh et al., 1990; Cloud et al., 1994; Russin et al., 1995). La enfermedad ocasiona la reducción del $30 \%$ en rendimiento y calidad del grano, y si el estrés persiste durante el desarrollo, las pérdidas pueden llegar al 100\% (Edmunds, 1964; MontesGarcía y Díaz-Franco, 2006).

La inoculación de las plantas de sorgo por medio de la técnica del palillo específica para M. phaseolina es ampliamente utilizada para inducir la enfermedad y así poder realizar comparaciones entre genotipos (Edmunds, 1964; Tuinstra et al; 2002). Por lo anterior, el objetivo del presente estudio fue evaluar la reacción a $M$. phaseolina de tres variedades experimentales y siete variedades de polinización libre del INIFAP, así como 14 híbridos comerciales de sorgo para identificar variedades e híbridos que puedan presentar tolerancia o menor susceptibilidad a esta enfermedad. 


\section{Método}

\section{Área de estudio}

El estudio se realizó en el municipio de Culiacán. Culiacán se ubica en la región central del Estado de Sinaloa, entre los meridianos $106^{\circ} 56^{\prime} 50^{\prime}$ " y $107^{\circ} 50^{\prime} 15^{\prime}$ " de longitud oeste del meridiano de Greenwich y las coordenadas extremas de los paralelos $24^{\circ} 02^{\prime} 10^{\prime \prime}$ y $25^{\circ} 14^{\prime} 56^{\prime \prime}$ de latitud norte. En Sinaloa, el 37,1\% de su superficie tiene un clima cálido subhúmedo con lluvias en verano, un 21,2\% presenta clima semiseco muy cálido y cálido, el 18,6\% seco muy cálido y cálido, un 11,0\% semicálido subhúmedo con lluvias en verano, y un 9,8\%, por un lado, y un $2.3 \%$, por otro, presentan otro tipo de climas; clima templado subhúmedo localizado en las partes altas de la Sierra Madre Occidental (INEGI, 2011).

Material genético. El material genético que se utilizó en cada experimento fueron siete variedades comerciales, generadas por el Instituto Nacional de Investigaciones Forestales, Agrícolas y Pecuarias (INIFAP), tres variedades experimentales, generadas en el Campo Experimental Valle de Culiacán (CEVACU) del INIFAP y 14 híbridos comerciales testigos.

\section{Inoculación}

La cepa de M. phaseolina utilizada en el estudio se obtuvo de plantas de sorgo infectadas de forma natural por el patógeno y colectadas en el ciclo Primavera-Verano 2009, bajo condiciones de temporal en el Campo Experimental Valle de Culiacán. La cepa se purificó en medio de cultivo papa-dextrosa-agar (PDA) fresco, acidificado con $1 \mathrm{ml}$ de ácido láctico L-1 y se incubó a $30{ }^{\circ} \mathrm{C}$ de cinco o siete días para el aislamiento.

\section{Tratamientos experimentales}

Los experimentos se establecieron durante los ciclos Primavera-Verano 2010 y 2011, bajo condiciones de campo en temporal en el Campo Experimental Valle de Culiacán del INIFAP en Culiacán, Sinaloa, México. El suelo es arcilloso pH 7.6 contenido bajo de N, medio P y alto de K. Se fertilizaron ambos experimentos con la fórmula 100-00-00. Se utilizó un diseño de bloques al azar en cada experimento con tres repeticiones y un análisis combinado para los dos ciclos agrícolas. La unidad experimental fue un surco de $5 \mathrm{~m}$ de largo y $0.80 \mathrm{~m}$ de separación. Se utilizó una densidad de población de 200, 000 plantas ha- ${ }^{1}$. En cada parcela experimental, las plantas 
fueron inoculadas artificialmente usando la técnica del palillo. Para reproducir el hongo se colocaron palillos de dientes estériles en cajas Petri con papa-dextrosa-agar (PDA) y la cepa del hongo. Se incubaron a $34^{\circ} \mathrm{C}$ durante 7 días, después se retiraron y secaron a temperatura ambiente. La inoculación de las plantas de sorgo, se realizó a los 21 días después del inició de la floración en 10 plantas por parcela (Edmunds, 1964; Williams-Alanís et al., 2004).

\section{Cuantificación de la severidad de la enfermedad}

De acuerdo al criterio establecido por Bramel-Cox (1988), se utilizó la longitud de lesión para cuantificar la severidad de la enfermedad ocasionada por el hongo Macrophomina phaseolina (Tassi) Goid al cultivo de sorgo en el estado de Sinaloa. En este estudio se consideró como tolerante a un genotipo cuando la longitud de la lesión presentó tamaños menores de 5.0 centimetros $(\mathrm{cm})$.

La evaluación de la longitud de lesión se realizó a los 21 días después de la inoculación. Los tallos se abrieron y se midió en $\mathrm{cm}$ la longitud de la lesión producida por el patógeno. Los datos de longitud de lesión fueron transformados con Log 10 para homogeneizar las varianzas y normalizar los datos. También se evaluó el rendimiento de grano por parcela, transformando a kg $\mathrm{ha}^{-1}$, y se estimó el coeficiente de correlación entre la longitud de lesión causada por $M$. phaseolina y el rendimiento de grano de los genotipos.

\section{Análisis estadístico}

Se realizó la comparación de medias (DMS p <0.05) para la diferenciación estadística de los genotipos. El análisis estadístico se llevó a cabo con el paquete estadístico SAS versión 9.1.3.

\section{Resultados y discusión}

Reacción de variedades experimentales y variedades de polinización libre e híbridos comerciales de sorgo a M. phaseolina.

El análisis de varianza detectó diferencias significativas $(\mathrm{p}<0.05)$ entre genotipos, longitud de lesión entre años y en la interacción genotipo años para severidad de lesión causada por M. phaseolina en sorgo, en Culiacán, Sinaloa en el ciclo P-V 2010 y en el análisis combinado (Cuadro 1). En el experimento establecido en el 2010, se observó una longitud de 
lesión promedio significativamente mayor $(10.10 \mathrm{~cm})$ en comparación con el sembrado en el agrícola $2011(5.79 \mathrm{~cm})$ (Cuadro 2).

Cuadro 1. Longitud de lesión y rendimiento de grano de sorgo de variedades e híbridos comerciales en Sinaloa, durante 2010 y 2011.

\begin{tabular}{llll}
\hline Fuentes de variación & GL & $\begin{array}{l}\text { CM } \\
\text { Longitud de lesión } \\
(\mathbf{c m})\end{array}$ & $\begin{array}{l}\text { CM } \\
\text { Rendimiento } \\
\left(\mathbf{k g ~ h a}^{-1}\right)\end{array}$ \\
\hline Genotipos (G) & 23 & $59.52^{*}$ & $1360784^{*}$ \\
Años (A) & 1 & $669.08^{*}$ & $421417^{*}$ \\
Repeticiones (R) & 2 & 0.75 & $187540^{*}$ \\
GXA & 23 & $47.99^{*}$ & 7061 \\
Error & & 0.06 & 15872 \\
\hline
\end{tabular}

Fuente: Elaboración propia. ${ }^{*}$ Significativo $(\mathrm{p} \leq 0.05)$

Las variedades de polinización libre y experimental del INIFAP más tolerantes durante los dos ciclos fueron Fortuna y Mazatlán-16, con una longitud de lesión de 3.5 y $4.95 \mathrm{~cm}$, respectivamente. A diferencia de las seis variedades de polinización libre que mostraron una longitud de lesión mayor a 5 cm: RB-Paloma (5.1 cm), Costeño-201 (5.2), Sinaloense-202 (5.25), Gavatero-203 (5.85 cm), Perla-101 $(6.5 \mathrm{~cm})$, Matinal $(6.75 \mathrm{~cm})$ y las dos variedades experimentales del INIFAP Dulce $(6.65 \mathrm{~cm})$ y Variedad-2 $(5.8 \mathrm{~cm})$, aunque resultaron más tolerantes que los híbridos comerciales, estas variedades no alcanzaron los niveles deseados de tolerancia $(<5.0 \mathrm{~cm}$ ) (Tenkouano et al., 1993; Pecina-Quintero et al., 1999). Con base en el análisis global de la reacción a $M$. phaseolina se pudo identificar que existen variedades de polinización libre del INIFAP con tolerancia al hongo que superan a híbridos comerciales testigos (Williams-Alanís, 1996; Pecina-Quintero et al., 1999). En el Cuadro 2 se presentan los resultados de las variedades experimentales y variedades de polinización libre por ciclo y en promedio de ambos ciclos agrícolas.

Los híbridos comerciales más afectados en los dos ciclos agrícolas por la pudrición carbonosa del tallo fueron Kilate; G-STAR-7402 y G-STAR-7205, con una longitud de lesión de 13.2, 15.7 y $16.65 \mathrm{~cm}$, respectivamente (Cuadro 3). 
Evaluación de la susceptibilidad a Macrophomina phaseolina (Tassi) Goid en diferentes variedades e híbridos comerciales de sorgo en Sinaloa, México

Cuadro 2. Longitud de lesión y rendimiento de grano en variedades experimentales y de polinización libre en Sinaloa, durante el 2010 y 2011.

\begin{tabular}{|c|c|c|c|c|c|c|}
\hline \multirow[t]{2}{*}{ Variedades } & \multirow{2}{*}{$\begin{array}{l}\text { Color de } \\
\text { grano }\end{array}$} & \multirow{2}{*}{$\begin{array}{l}\text { Ciclo } \\
\text { vegetativo }\end{array}$} & \multicolumn{2}{|c|}{$\begin{array}{l}\text { Longitud de lesión } \\
\text { por ciclo }(\mathrm{cm})\end{array}$} & \multirow{2}{*}{$\begin{array}{l}\text { Longitud } \\
\text { de lesión } \\
\text { promedio } \\
\text { (cm) }\end{array}$} & \multirow{2}{*}{$\begin{array}{l}\text { Rend. } \\
\text { promedio } \\
\text { grano } \\
\left(\text { kg ha }^{-1}\right)\end{array}$} \\
\hline & & & 2010 & 2011 & & \\
\hline Mazatlán-16* & Crema & Intermedio & 5.4 & 4.5 & 4.95 & 3253 \\
\hline Variedad-2* & Crema & Intermedio & 5.4 & 6.2 & 5.8 & 2709 \\
\hline Dulce* & Crema & Intermedio & 6.8 & 6.5 & 6.65 & 2021 \\
\hline Promedio & & & 5.87 & 5.73 & 5.80 & 2661 \\
\hline Fortuna+ & Crema & Tardío & 3.4 & 3.6 & 3.5 & 1901 \\
\hline RB-Paloma+ & Crema & Intermedio & 4.8 & 5.4 & 5.1 & 1913 \\
\hline Costeño-201+ & Crema & Intermedio & 4.8 & 5.6 & 5.2 & 3350 \\
\hline Sinaloense-202+ & Crema & Intermedio & 5.7 & 4.8 & 5.25 & 2810 \\
\hline Gavatero-203+ & Rojo & Intermedio & 7.5 & 4.2 & 5.85 & 2719 \\
\hline Perla-101+ & Crema & Int-precoz & 6.1 & 6.9 & 6.5 & 2529 \\
\hline Matinal+ & Crema & Precoz & 8.1 & 5.4 & 6.75 & 2485 \\
\hline Promedio & & & 5.77 & 5.13 & 5.45 & 2530 \\
\hline DMS $(\mathrm{p} \leq 0.05)$ & & & 0.502 & 0.337 & 0.224 & 186.4 \\
\hline
\end{tabular}

Fuente: Elaboración propia. $*$ = variedades experimentales;

$+=$ variedades de polinización libre.

Los datos promedio de rendimiento en grano muestran que 10 testigos comerciales tienen rendimientos mayores a los 3000 kg ha- ${ }^{1}$ (Cuadro 3).

Cuadro 3. Longitud de lesión y rendimiento de grano en híbridos comerciales de sorgo en Sinaloa, durante el 2010 y 2011.

\begin{tabular}{|c|c|c|c|c|c|c|}
\hline \multirow[t]{2}{*}{ Hibridos comerciales } & \multirow{2}{*}{$\begin{array}{l}\text { Color de } \\
\text { grano }\end{array}$} & \multirow{2}{*}{$\begin{array}{c}\text { Ciclo } \\
\text { vegetativo }\end{array}$} & \multicolumn{2}{|c|}{$\begin{array}{c}\text { Longitud de lesión } \\
\text { por ciclo }(\mathrm{cm})\end{array}$} & \multirow{2}{*}{$\begin{array}{l}\text { Longitu } \\
\text { d de } \\
\text { lesión } \\
\text { promed } \\
\text { io }(\mathrm{cm})\end{array}$} & \multirow{2}{*}{$\begin{array}{c}\text { Rend. } \\
\text { Pro- } \\
\text { medio } \\
\text { grano } \\
\left(\mathrm{kg}^{-}\right. \\
\left.\text {ha }^{-1}\right)\end{array}$} \\
\hline & & & 2010 & 2011 & & \\
\hline G-STAR-7304 (T) & Rojo & Intermedio & 8.2 & 5.7 & 6.95 & 3337 \\
\hline G-STAR-7601 (T) & Rojo & Intermedio & 8.5 & 5.9 & 7.2 & 3501 \\
\hline
\end{tabular}




\begin{tabular}{lllllll}
\hline P-8641 (T) & Rojo & Intermedio & 8.7 & 6.5 & 7.6 & 3033 \\
AVANTE MESQUITE (T) & Rojo & Intermedio & 9.4 & 6.1 & 7.75 & 2819 \\
AVANTE NOGAL (T) & Rojo & Intermedio & 10.9 & 5.3 & 8.1 & 3092 \\
AVANTE CIPRES (T) & Rojo & Intermedio & 9.2 & 7.1 & 8.15 & 3003 \\
SYNGENTA 5515 (T) & Rojo & Intermedio & 11.3 & 5.2 & 8.25 & 3154 \\
86P42 (T) & Rojo & Intermedio & 10.8 & 5.9 & 8.35 & 3458 \\
AVANTE PINO (T) & Rojo & Intermedio & 11.6 & 5.4 & 8.5 & 3272 \\
SYNGENTA 5265 (T) & Rojo & Intermedio & 11.0 & 6.3 & 8.65 & 3428 \\
85P47 (T) & Rojo & Intermedio & 13.7 & 6.7 & 10.2 & 2931 \\
KILATE (T) & Rojo & Intermedio & 20.5 & 5.9 & 13.20 & 2962 \\
G-STAR-7402 (T) & Rojo & Intermedio & 24.6 & 6.8 & 15.70 & 2986 \\
G-STAR-7205 (T) & Rojo & Intermedio & 26.1 & 7.2 & 16.65 & 3359 \\
Promedio & & & $\mathbf{1 3 . 1 8}$ & $\mathbf{6 . 1 4}$ & $\mathbf{9 . 6 6}$ & $\mathbf{3 1 6 6}$ \\
DMS (p $\leq 0.05)$ & & & 0.575 & 0.402 & 0.212 & 184.4 \\
& & & & & 9 \\
\hline
\end{tabular}

Fuente: Elaboración propia.

Los hibridos comerciales superan en rendimiento promedio de grano a una variedad experimental (variedad-2) y a seis variedades de polinización libre (Fortuna, RB-Paloma, Sinaloense-202, Gavatero-203, Perla-101 y Matinal). No obstante, la variedad Costeño-201 y la variedad experimental Mazatlán-16 compitieron favorablemente respecto a los híbridos comerciales al obtener 3350 y $3253 \mathrm{Kg} \mathrm{ha-}^{1}$, respectivamente, a un nivel de DMS del 5\%.

Aunque los híbridos comerciales presentaron en promedio $20.08 \%$ mayor rendimiento de grano respecto a las variedades de polinización libre, fueron más susceptibles al patógeno (9.66 $\mathrm{cm}$ en promedio) que las variedades experimentales $(5.80 \mathrm{~cm}$ promedio) y las variedades de polinización libre (5.45 cm promedio) del INIFAP. El coeficiente de correlación de Pearson entre la longitud de lesión y el rendimiento de grano de variedades e híbridos de sorgo fue de 0.023 indicando ausencia de correlación de Pearson, similar a lo reportado por Williams-Alanís, et al. (2009).

En los experimentos de sorgo durante los ciclos Primavera-Verano 2010 y 2011, bajo condiciones de campo en temporal, no se encontró un efecto directo entre $M$. phaseolina con el 
rendimiento de grano. Sin embargo, se observó durante varios ciclos en Primavera-Verano bajo condiciones de campo en temporal en Sinaloa que cientos de hectáreas de sorgo son afectadas por M. phaseolina causando el acame de las plantas, imposibilitando la cosecha mecánica y la pérdida en el rendimiento de grano. Estudios realizados en maíz han reportado una situación similar, lo cual sugiere que la perdida mayor en el rendimiento de cultivo provocado por la enfermedad se deba más al acame de la planta que al efecto directo del hongo en el grano (DíazFranco et al., 2008). Lo anterior es provocado por la destrucción parcial o total de los tejidos en la base del tallo por el patógeno, lo que impide la translocación de nutrientes hacia la panoja para la formación y llenado de grano (Tunistra et al., 2002).

En estudios anteriores, Williams-Alanís et al. (2009) obtuvieron la reacción de las variedades de sorgo para grano a M. phaseolina, la severidad de lesión encontradas en variedades de polinización libre fueron las siguientes: S-23 (Sinaloense-202) $(15.5 \mathrm{~cm})$, Perla-101 $(23.2 \mathrm{~cm})$, Costeño-201 (14.2 cm), Var-9 (RB-paloma) $(19.6 \mathrm{~cm})$ y Amarillo precoz (Matinal) $(9.2 \mathrm{~cm})$. En las variedades experimentales M-16 (Mazatlán-16) $(21.6 \mathrm{~cm})$ y Dulce $(12.1 \mathrm{~cm})$, lo cual difiere a los resultados obtenidos en el presente estudio, la severidad de lesión en variedades comerciales S-23 (Sinaloense-202) (5.25 cm), Perla-101 (6.5 cm), Costeño-201 (5.2 cm), Var-9 (RB-paloma) $(5.1 \mathrm{~cm})$ y Amarillo precoz (Matinal) (6.75) y en las variedades experimentales M-16 (Mazatlán16) $(4.95 \mathrm{~cm})$ y Dulce $(6.65 \mathrm{~cm})$ respectivamente, resultando la variedad de polinización libre Matinal muy similar en la longitud de lesión a lo reportado por Williams-Alanís et al. (2009). Estos mismos autores, consideraron a la variedad Amarillo precoz (Matinal) como más tolerante a M. phaseolina y para la formación de progenitores e híbridos tolerantes en Tamaulipas, México. Las diferencias obtenidas en la severidad de lesión en el presente estudio y lo reportado por Williams-Alanís et al. (2009) se pueden atribuir a diferentes factores tales como: condiciones climáticas, adaptación, sequía, prácticas agronómicas y patogenicidad de cepas o aislamientos del patógeno M. phaseolina en sorgo.

Dhingra y Sinclair (1978) encontraron variación en la virulencia entre aislados colectados de una misma planta de soya. Algo similar se observó al examinar aislados colectados de un mismo hospedero (sorgo o algodón) (Diourte et al., 1995; Sulaiman y Patil, 1996). Estudios realizados en dos sistemas de marcadores moleculares; Polimorfismos del DNA amplificado al azar (RAPDs) y Polimorfismos en la longitud de los fragmentos amplificados (AFLPs) han 
demostrado la existencia de una gran diversidad genética en esta especie (Jiang, 1995; PecinaQuintero et al., 2001).

Williams-Alanís et al. (2009) reportan los siguientes rendimientos promedio en variedades de polinización libre S-23 (Sinaloense-202) (1620 kg ha'-1), Perla-101 (2112 kg ha-1), Costeño-201 (1998 kg ha-1), Var-9 (RB-paloma) (2113 kg ha-1) y Amarillo precoz (Matinal) (1994 kg ha $\mathrm{kg}^{-1}$ y en las variedades experimentales M-16 (Mazatlán-16) (2389 kg ha-1) y Dulce (2429 $\left.\mathrm{kg} \mathrm{ha}^{-1}\right)$, lo cual difiere con los resultados obtenidos en el presente estudio. El rendimiento promedio en variedades de polinización libre S-23 (Sinaloense-202) (2810 kg ha-1), Perla-101 (2529 $\left.\mathrm{kg} \mathrm{ha}^{-1}\right)$, Costeño-201 (3350 kg ha-1), Var-9 (RB-paloma) (1913 kg ha ${ }^{-1}$ ) y Amarillo precoz (Matinal) (2485 kg ha-1) y en las variedades experimentales M-16 (Mazatlán-16) (3253 kg ha-1) y Dulce $\left(2021 \mathrm{~kg} \mathrm{ha}^{-1}\right)$, respectivamente. Se ha reportado que las diferencias entre los resultados de rendimiento de grano se deben a varios factores como diferentes condiciones climáticas, sequía, precipitación pluvial, temperatura, fechas de siembra y adaptación (Singh et al., 1990; Cloud et al., 1994; Russin et al., 1995).

\section{Conclusiones}

En conclusión, se identificaron dos genotipos menos susceptibles a la pudrición carbonosa del tallo causada por M. phaseolina: Fortuna y Mazatlán-16 (3.5 y 4.95 centimetros de longitud de lesión promedio), por lo cual se sugieren para siembras de temporal y como progenitores en la formación de híbridos de sorgo tolerantes a esta enfermedad en el Estado de Sinaloa, México.

Por otro lado, es importante señalar que la variedad Costeño-201 presentó una longitud promedio de lesión de 5.2 centimetros, y junto con la variedad experimental Mazatlán-16 compiten favorablemente en rendimiento respecto a los híbridos comerciales, al obtener 3350 y $3253 \mathrm{Kg} \mathrm{ha}^{-1}$ respectivamente, en comparación con los $3166 \mathrm{Kg} \mathrm{ha}^{-1}$ promedio de los 14 tipos de hibridos evaluados, a un nivel de DMS del $5 \%$.

Finalmente, de acuerdo con los resultados obtenidos en el presente estudio no existe correlación entre la longitud de lesión causada $M$. phaseolina y el rendimiento de grano de variedades e híbridos de sorgo en el estado de Sinaloa. 
Evaluación de la susceptibilidad a Macrophomina phaseolina (Tassi) Goid en diferentes variedades e híbridos comerciales de sorgo en Sinaloa, México

\section{Agradecimientos}

Al apoyo financiero proporcionado por Fundación Produce Sinaloa, A. C. a través del proyecto. No. 2055985A titulado: Formación de variedades e híbridos de sorgo para temporal y riego en el estado de Sinaloa.

\section{Referencias}

Bramel-Cox, P., S. Stein, D. Rodgers and L. Claflin (1988). Inheritance of resistance to Macrophomina phaseolina (Tassi) Goid and Fusarium moniliforme Sheldon in sorghum. Crop Science 28: 37-40.

Clafin, L. E. and L. M. Giorda (2002). Stalk rots of sorghum. In: J.F. Leslie (ed.). Sorghum and Millet Diseases. Chapter 33. Iowa State Press. Ames, USA. pp. 185-190.

Cloud, G. and J. Rupe (1994). Influence of nitrogen, plant growth stage and environment on charcoal rot of grain sorghum caused by Macrophomina phaseolina (Tassi) Goid. Plant and Soil 158: 203-210.

Cook, G., M. G. Boosalis, L. Dunkle and C. Odvody (1973). Survival of Macrophomina phaseolina in corn and sorghum stalk residue. Plant Disease Report 57: 873-874.

Dhingra, O.D., and J. B. Sinclair (1978). Biology and Pathology of Macrophomina phaseolina. Universidade Federal de Vicosa. Vicosa, Brazil. 166 p.

Díaz-Franco, A., J. R. Salinas-García, I. Garza-Cano, y N. Mayek-Pérez (2008). Impacto de labranza e inoculación mycorrízica arbuscular sobre la pudrición carbonosa del tallo y rendimiento de maíz en condiciones semiáridas. Revista Fitotecnia Mexicana 31:257-263.

Diourte, M., J. L. Starr, Jeger, M. J. Stack, J.P. and D. T. Rosenow (1995). Charcoal rot (Macrophomina phaseolina) resistance and effects of water stress on disease development in sorghum. Plant Pathology 44:196-202.

No 18, Vol. 9 (1), 2017. ISSN 2007 - 0705, pp.: 186 - 199 
Edmunds, L.K. (1964). Combined relation of plant maturity, temperature, and soil moisture to charcoal stalk rot development in grain sorghum. Phytopathology 54:514-517.

Jiang, Y. (1995). Genetic variation among Macrophomina phaseolina isolates as detected by PCR-based techniques. MS Thesis. South Dakota State University, Pierre, South Dakota, USA. 55 p.

INEGI. (2011). Perspectiva Estadística Sinaloa. [En línea]. Disponible en: http://www.inegi.org.mx/est/contenidos/espanol/sistemas/perspectivas/perspectiva-sin.pdf (17 mayo 2014)

Montes-García, N. and Díaz-Franco, A. (2006). Fitopatología. pp. 192-213. En: L.A. Rodríguez del Bosque (ed.). Campo Experimental Río Bravo: 50 Años de Investigación Agropecuaria en el Norte de Tamaulipas, Historia, Logros y Retos. Capítulo 12. Libro Técnico No. 1. INIFAP, Campo Experimental Río Bravo. Río Bravo, Tamaulipas, México. 325p.

Mihail, J. D. and S. J. Taylor (1995). Interpreting variability among isolates of Macrophomina phaseolina in pathogenicity, pycnidium production, and chorate utilization. Canadian Journal of Botany 73:1596-1603.

Olaya, G., G. S. Abawi, and N. F. Weeden, (1996). Inheritance of the resistance to Macrophomina phaseolina and identification of RAPD markers linked to the resistance genes in beans. Phytopathology 86:674-679.

Pecina-Quintero, V., H. Williams-Alanís, and G. J. Vandemark (1999). Diallel analysis of resistance to Macrophomina phaseolina in sorghum. Cereal Research Communications 27:99-106.

Pecina-Quintero, V., V. O. Martínez, B. M. J. Alvarado, G. J. Vandemark and H. WilliamsAlanís (2001). Comparación de dos sistemas de marcadores moleculares en el análisis de 
Evaluación de la susceptibilidad a Macrophomina phaseolina (Tassi) Goid en diferentes variedades e híbridos comerciales de sorgo en Sinaloa, México

las relaciones genéticas de Macrophomina phaseolina (Tassi) Goid. Revista Mexicana de Fitopatología, A.C. 19 (002): 128-139.

Russin, J., Carter and J. Griffin (1995). Effects of grain sorghum herbicides on charcoal rot fungus. Weed Technology. 9:343-351.

SAS Institute Inc., SAS 9.1.3. (2000-2004) Help and Documentation, Cary, NC: SAS Institute Inc.

Servicio de Información y Estadística Agroalimentaria y Pesquera (SIAP). 2016. Anuario estadístico de la producción agrícola 2014 en México. El cultivo de sorgo. SAGARPA. URL: http://www.siap.gob.mx. (15 de agosto de 2015)

Singh, S. K., Y. L. Nene and M. V. Reddy (1990). Influence of cropping systems on Macrophomina phaseolina populations in soil. Plant Disease. 74:812-814.

Sulaiman, M. and B. C. Patil (1996). Existence of physiological races of Macrophomina phaseolina causing root rot of cotton. Beir Trop. Subtrop. Landwirtsch Tropenveterinaermed. 4:291-298.

Tenkouano, A., F. R. Millar, R. A. Frederiksen, and D. T. Rosenow (1993). Genetics of no senescence and charcoal rot resistance in sorghum. Theoretical and Applied Genetics 85:644-648.

Tunistra, M.R., T. T. Teferra, L. E. Claflin, R. G. Henzell, A. Borrell, N. Seetharama, G. Ejeta and D. T. Rosenow (2002). Breeding for resistance to root and stalk rots in sorghum. In: J.F. Leslie (ed.). Sorghum and Millet Diseases. Chapter 33. Iowa State Press. Ames, USA. pp. 281-286. 
Williams-Alanís, H. (1996). RB-4040, nuevo híbrido de sorgo para el noreste de México y tolerante a Sporisorium reilianum y Macrophomina phaseolina. Revista Fitotecnia Mexicana 19: 193-194.

Williams-Alanís, H., F. Zavala-García, R. Martínez-Hernández, S. E. Rangel-Estrada, and I. Machuca-Orta (2004). Reacción a Macrophomina phaseolina (Tassi) Goid de híbridos comerciales y experimentales de Sorgo [Sorghum bicolor (L.) Moench.] para grano. Revista Mexicana de Fitopatología 22:216-222.

Williams-Alanís, H., V. Pecina-Quintero, N. Montes-García, O. Palacios-Velarde, G. ArcosCavazos, and V. A. Vidal-Martínez (2009). Reacción de variedades de sorgo [Sorghum bicolor (L.) Moench.] para grano a Macrophomina phaseolina (Tassi) Goid. Rev. Mex. Fitopatol. 27:148-155. 